\title{
étude expérimentale de la sollicitation exercée par le sol sur les ouvrages de soutènement souples
}

\author{
P. SCHMITT \\ Ingénieur chef de projet - Service Technique et Développement \\ Soletanche-entreprise*
}

\section{INTRODUCTION}

L'Ingénieur confronté au calcul d'un ouvrage de soutènement souple (rideau de palplanches ou paroi en béton armé, moulée ou préfabriquée) doit, en premier lieu, définir les efforts exercés par le terrain sur l'écran.

Or, ceux-ci dépendent non seulement du sol mais encore, dans une large mesure, des dimensions de l'ouvrage et des possibilités qu'il a de se déplacer. Les méthodes classiques s'affranchissent de cette difficulté en supposant les déplacements suffisamment importants pour que les massifs de sol entourant l'écran atteignent l'état d'équilibre-limite. Des méthodes plus récentes (méthode dite "élastoplastique» ou méthode des éléments finis) tiennent compte d'un comportement élastique du sol pour de plus faibles déplacements; elles sont donc plus générales, mais nécessitent la connaissance d'un plus grand nombre de paramètres.

En particulier la méthode «élastoplastique », fort répandue en pratique, repose sur la notion de *coefficient de réaction élastique» du sol, paramètre de nature empirique dont la détermination n'est donc possible qu'à partir d'un large support expérimental.

Il nous a semblé intéressant, dans cette optique, de regrouper un ensemble de résultats expérimentaux récents, obtenus sur des sites divers, permettant de mieux approcher le comportement réel de l'interface terrain-écran.

(*) 6, rue de Watford - 92000 Nanterre.

\section{RAPPEL DE QUELQUES RÉSULTATS CLASSIQUES}

La sollicitation exercée par le sol sur les ouvrages de soutènement est classiquement définie par le diagramme de la figure $\mathrm{n}^{\circ} 1$ déduit des expériences de Terzaghi et Rowe, citées par Caquot et Kérisel (réf. 4), et consistant à déplacer des écrans rigides dans des sols homogènes.

Ce diagramme montre:

- que la poussée minimale, ou poussée limite, est atteinte pour de très faibles déplacements (quelques \%。 de la hauteur de l'écran), ce qui tend à justifier les méthodes de calcul classiques;

- que la butée maximale, ou butée limite, n'est atteinte, au contraire, que pour de grands déplacements (quelques \% de la hauteur de l'écran) généralement incompatibles avec la rigidité du butonnage ou la précontrainte exercée par les tirants.

La sollicitation exercée par le sol sur l'écran se décompose donc schématiquement en poussée limite, calculable à partir des théories classiques, et «butée élastique ».

En pratique on substitue au diagramme de la figure $n^{\circ} 1$ celui de la figure $n^{\circ} 2$, dans lequel le comportement élastique du sol est entièrement linéarisé.

Cette schématisation permet de caractériser le comportement élastique du terrain par un paramètre unique $k$, appelé «coefficient de réaction » et défini comme étant 


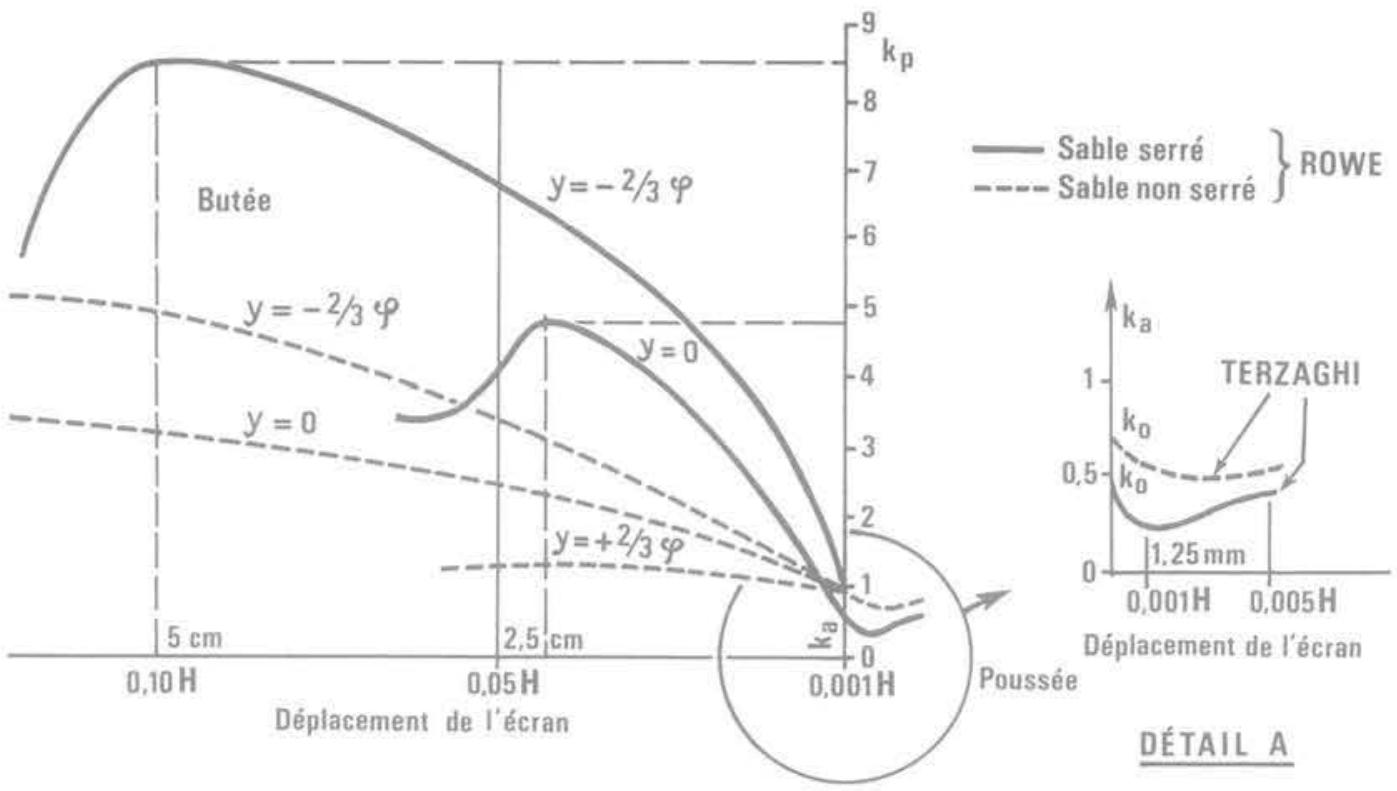

Fig. 1. - Sollicitation exercée par le sol sur un écran vertical rigide de hauteur $H$.

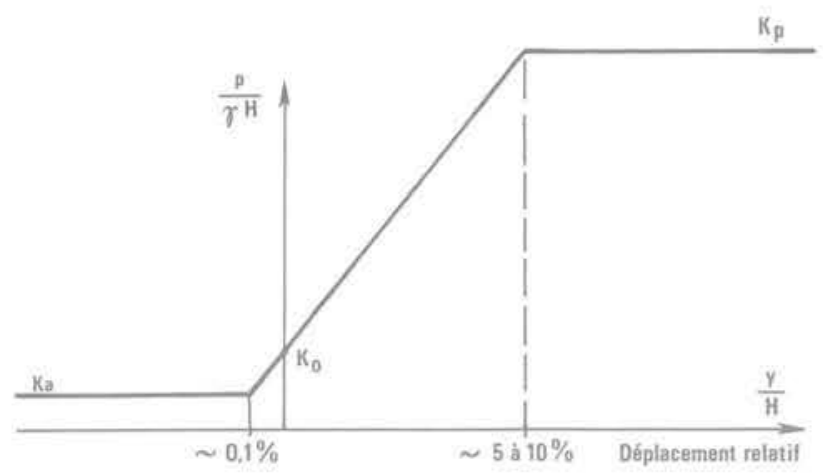

Fig. 2. - Schéma habituellement adopté pour le calcul élastoplastique.

le rapport, supposé constant, de la variation de pression au déplacement associé en tout point de l'interface sol-structure. Il s'agit en fait d'un artifice de calcul sans grand fondement théorique, le coefficient $k$ ayant notamment la prétention de représenter, à lui seul, les coefficients d'élasticité du sol ainsi que l'ensemble des conditions aux limites.

Les méthodes traditionnelles d'évaluation du coefficient de réaction sont les suivantes:

- Utilisation des ordres de grandeur recommandés par Terzaghi (réf. 3 et 13).

- Utilisation de la formule de Ménard et Bourdon (réf. 10), qui proposent d'évaluer le coefficient de réaction $k_{D}$ du sol en butée contre la fiche d'un rideau en supposant l'existence d'un centre de rotation situé à la profondeur $\mathrm{D}=2 / 3 \times \mathrm{H}$, comme indiqué sur la figure $n^{\circ} 3$, et en écrivant:
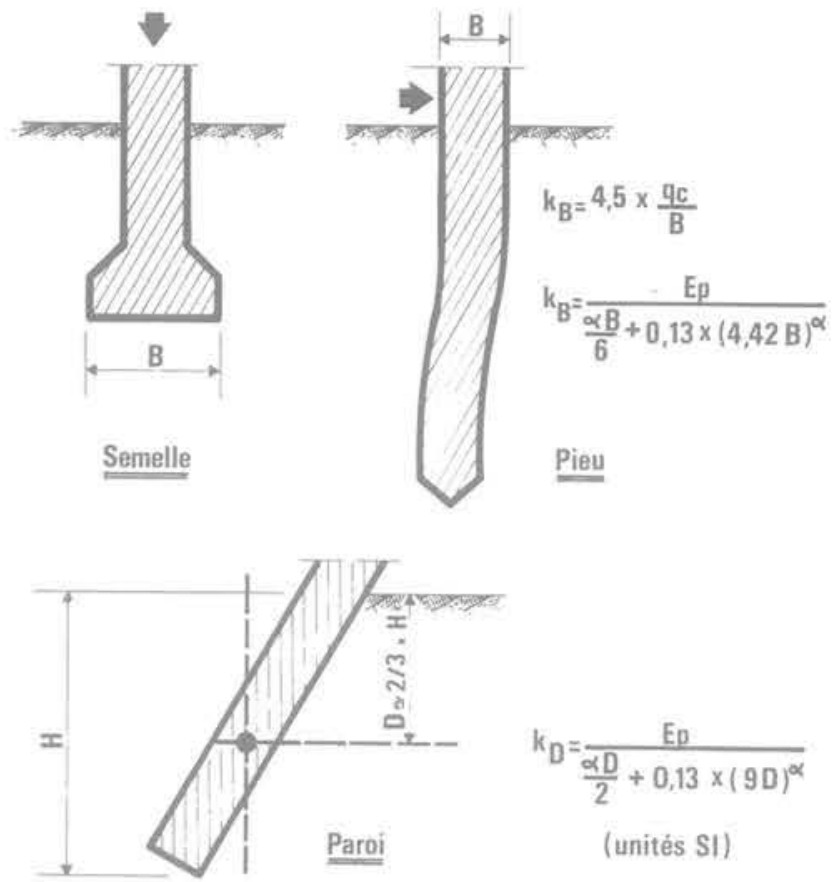

Fig. 3. - Expressions du coefficient de réaction.

$$
k_{D}=\frac{E p}{\frac{\alpha D}{2}+0.13 \times(9 D)^{\alpha}}
$$

Ep étant le module pressiométrique et $\alpha$ le «coefficient de structure " du sol.

Pour ce qui concerne la réaction élastique du sol en contre-butée, Terzaghi et Ménard (rếf. 10 et 13) 
indiquent tous deux des valeurs sensiblement plus importantes qu'en butée.

Des formules analogues ont par ailleurs été proposées pour traiter le problème des fondations telles que semelles filantes ou pieux sollicités horizontalement:

$$
k_{B}=\frac{E p}{\frac{\alpha B}{6}+0.13 \times(4.42 B)^{\alpha}}
$$

formule proposée par Mênard (réf. 11),

B étant la largeur de la fondation, comme indiqué sur la fig. $\mathrm{n}^{\circ} 3$,

$$
k_{B}=\frac{4.5 \times q c}{B}
$$

formule proposée par Lacroix, (rêf. 8),

qc étant la résistance de pointe mesurée au pénêtromètre statique.

On constate que ces différentes formules font systématiquement intervenir un ou deux paramètres caractérisant la compressibilité du sol et un paramètre représentant la dimension de l'aire comprimée.

\section{PRINCIPE DE L'ÉTUDE}

Compte tenu du caractère extrêmement empirique des méthodes évoquées ci-dessus, leur application aux ouvrages de soutènement souples de grandes dimensions mérite, chaque fois que cela est possible, qu'il soit procédé à des vérifications expérimentales sur ouvrages réels, même si les mesures effectuées sur chantier, dans des conditions souvent rudimentaires, ne permettent d'obtenir que des ordres de grandeur.

Nous avons, dans cette optique, envisagé deux approches successives:

- une première approche, que l'on peut qualifier de "locale», consiste à tirer parti d'un essai couramment pratiqué sur les chantiers, qui est l'essai de mise en tension des tirants forés précontraints par la méthode du cycle. Cet essai consiste à mesurer, au moyen d'un comparateur lié à une base fixe, l'allongement puis le raccourcissement du tirant au cours d'un cycle de chargement, exercé au moyen d'un vérin prenant appui sur la paroi ou le rideau. Chaque mise en tension constitue donc un véritable essai de plaque sur l'ouvrage lui-même; il suffit dès lors de mesurer également le déplacement horizontal de la paroi au cours du cycle de chargement pour obtenir une courbe expérimentale caractérisant le comportement local de l'ouvrage.

- une seconde approche, plus « globale», consiste à mesurer, au moyen de tubes inclinométriques et de relevés topographiques, la déformation de l'ouvrage sur toute la hauteur au cours des différentes phases de travaux.

Ces deux approches complémentaires permettent de caractériser in situ la compressibilité du terrain.

\section{APPROCHE «LOCALE»}

On trouvera sur les figures $n^{\circ} 4$ à 7 quelques exemples de courbes effort-déplacement obtenues sur différents chantiers lors des cycles de mise en tension, intégrant systêmatiquement un ou plusieurs paliers de fluage.

$$
\text { St. PRIX - Parôt mouláo }
$$

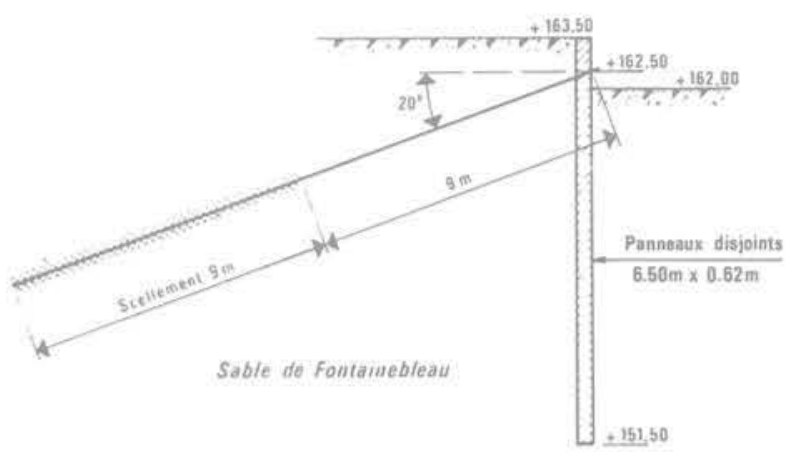

St. PRIX - DEPIACEMENT DE LA PAROI

Essai du $16-12-75$. Tirant 6 B

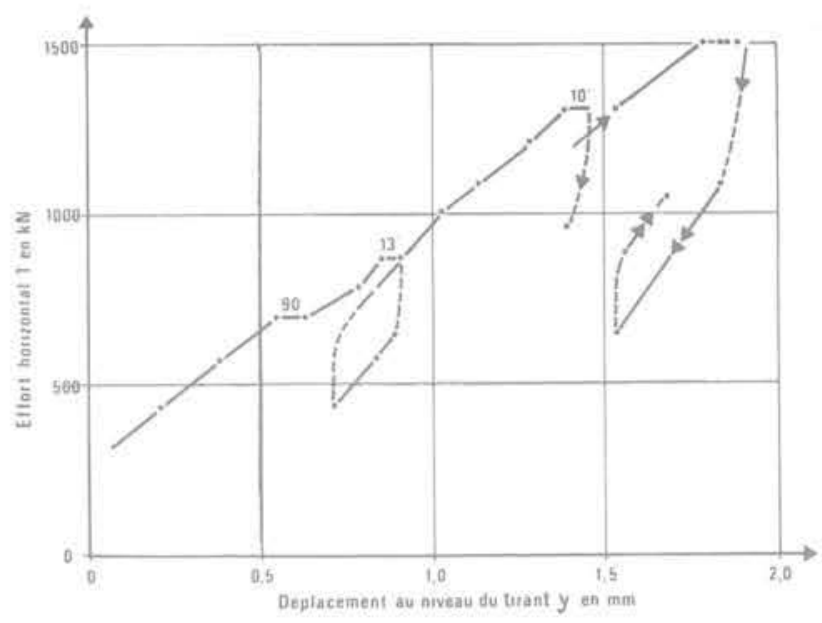

Fig. 4. - St-Prix - Essai de chargement horizontal.

Le comportement observé n'est généralement ni parfaitement élastique (le déplacement résiduel pouvant atteindre le tiers du déplacement total) ni parfaitement linéaire (surtout dans le domaine des déplacements inférieurs au $\mathrm{mm}$ ). Ces observations semblent donc confirmer l'hypothèse d'un module supérieur pour les «micro-déformations du sol», comme indiqué par Terzaghi et Ménard (réf. 9 et 13).

II est possible, en utilisant la théorie des poutres sur appuis élastiques infiniment rapprochés (réf. 5 et 
MUR DE QUAI - DEAUVILLE

Paroi préfabriquée

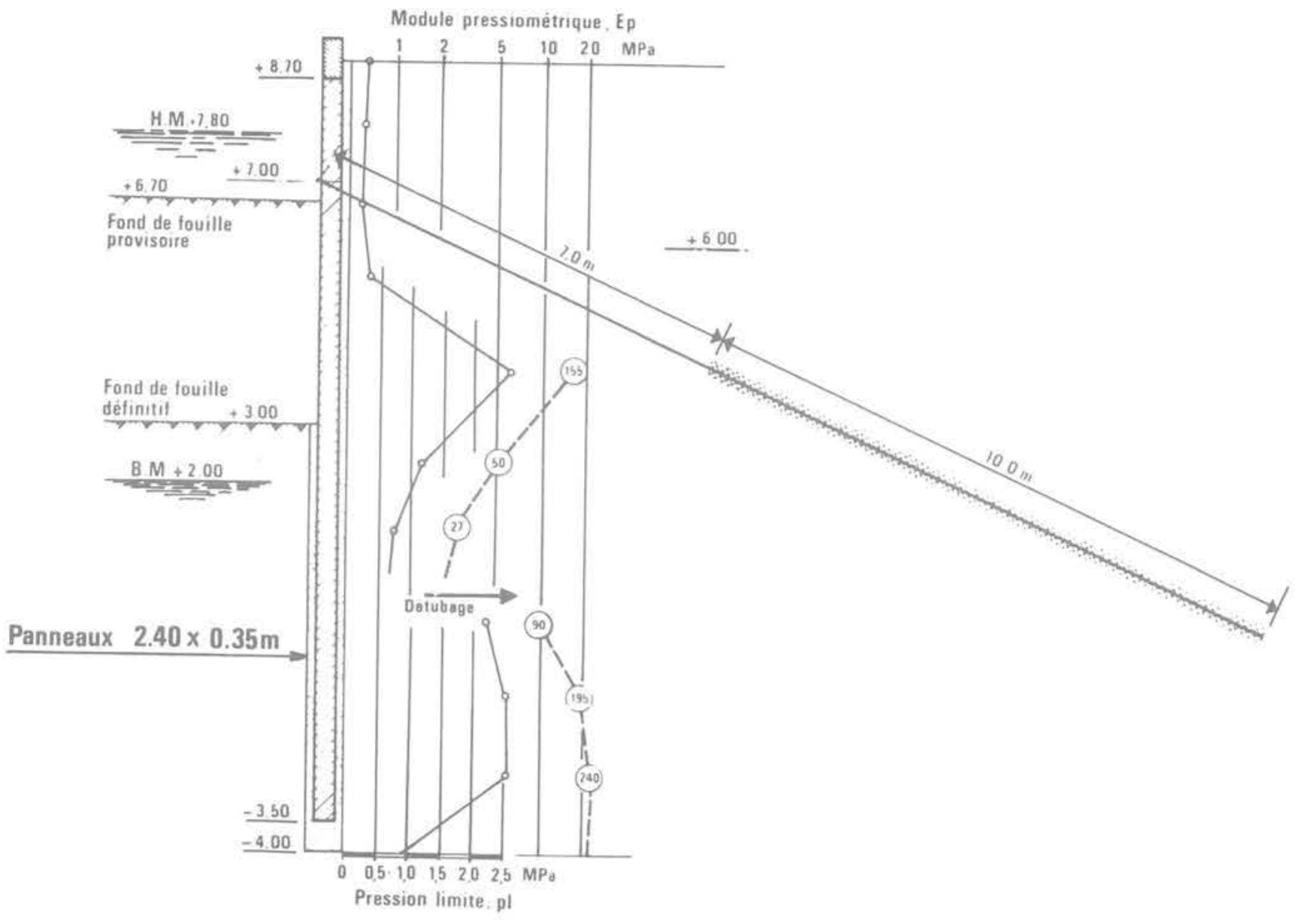

DEAUVILLE

Deplacements au niveau du tirant

-...- Comparateur nч1

Comparateur n 2

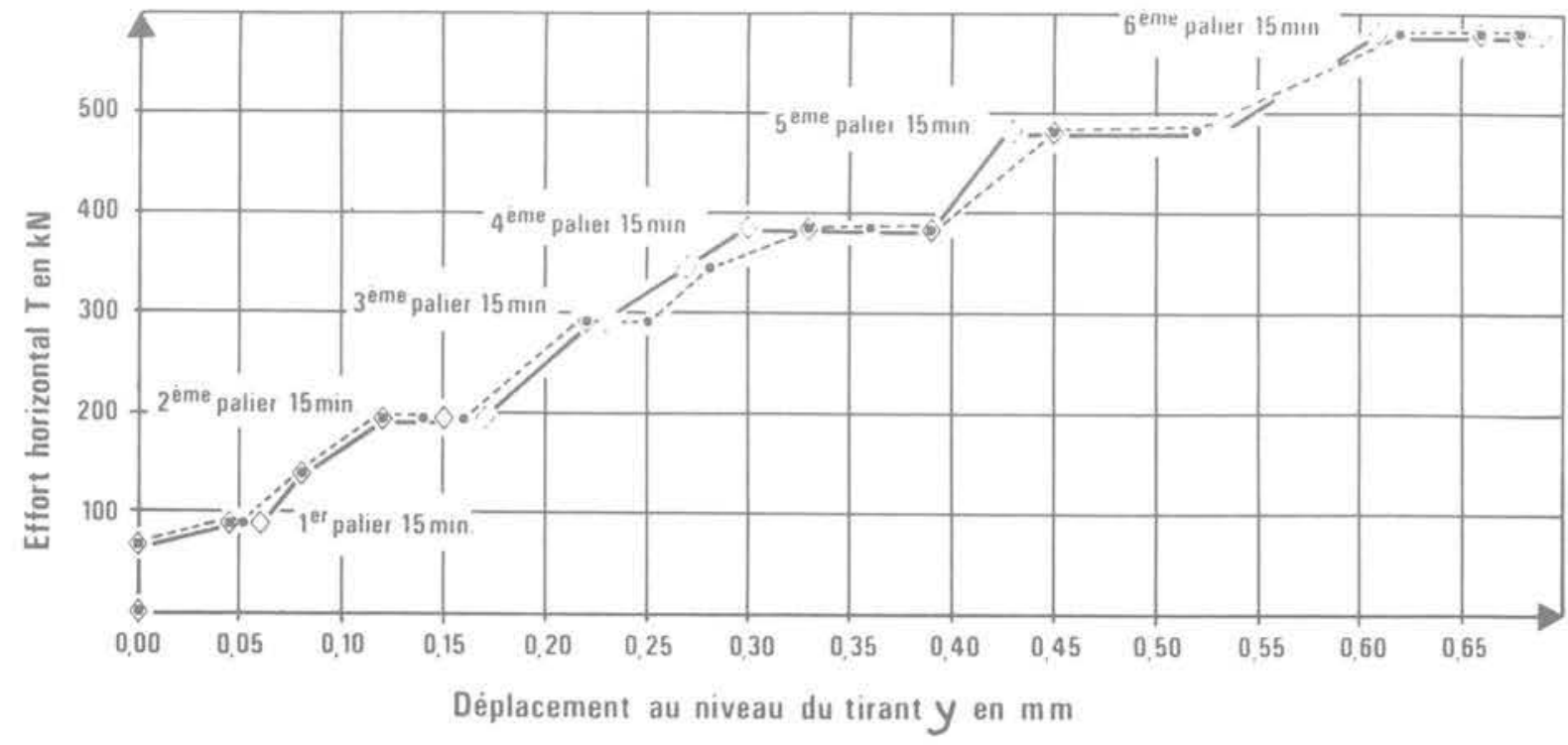

Fig. 5. - Deauville - Essai de chargement horizontal. 


\section{Métro de Lille - Paroi Préfabriquée}

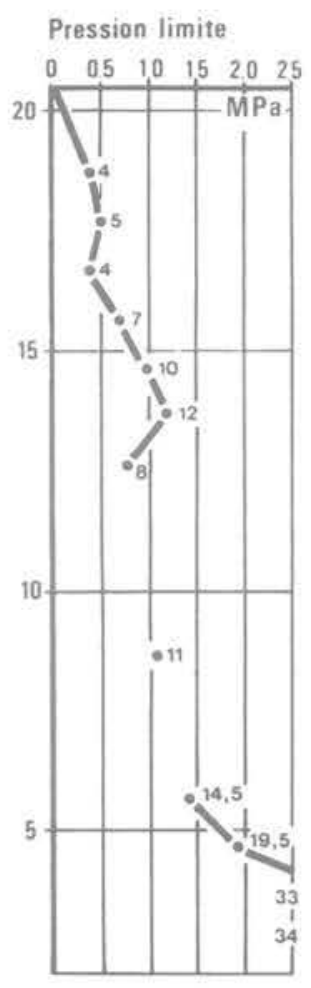

Module pressiometrique Résistance de pointe
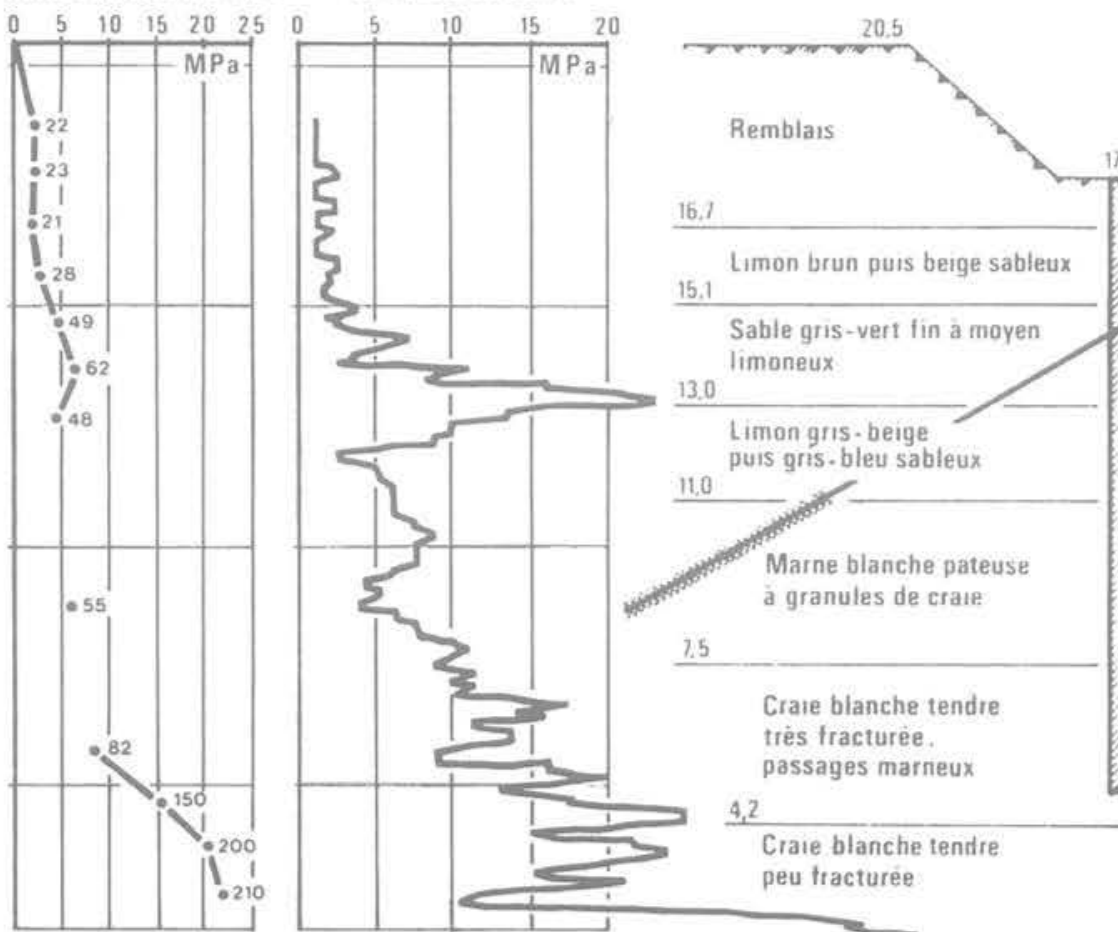

Limon brun puis beige sableux

Sable gris-vert fin à moyen

3.0 Limon gris, heige puis gris. bleu sableux

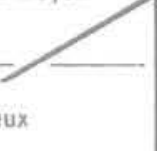
11,0

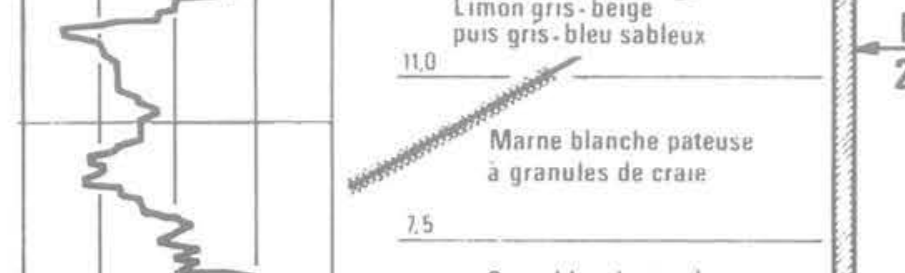

Panneaux $2.00 \times 0.50 \mathrm{~m}$

\section{LILLE . DEPLACEMENT DE LA PAROI \\ ESSAI DU 7.11 .79 \\ TIRANT 112}

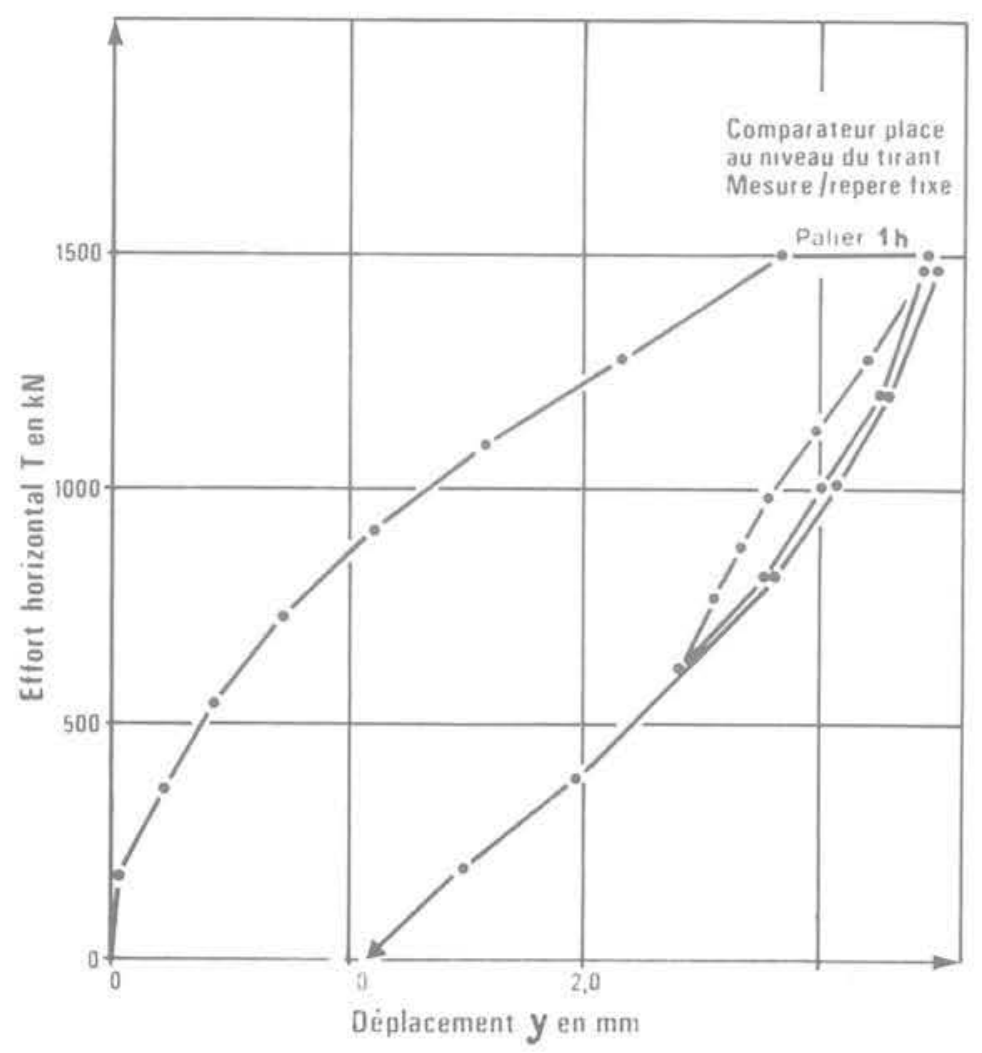

Fig. 6. - Lille - Essai de chargement horizontal. 


\section{- METRO DE LILLE-PAROI PREFABRIOUEE-}

Dispositif de mesures

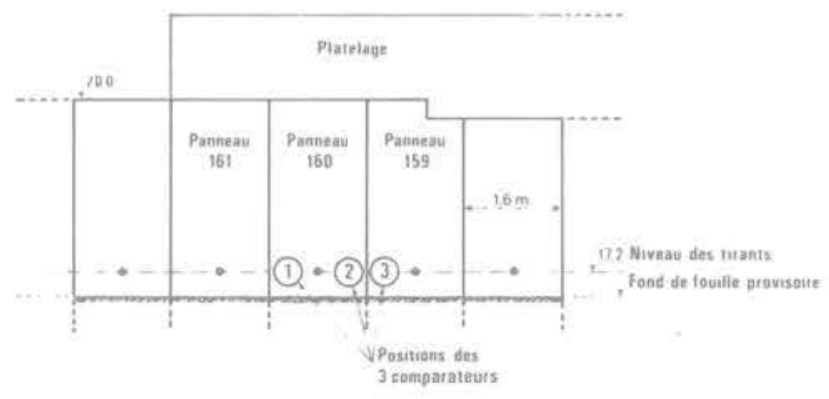

LILL DEPLACEMENT DE LA PAROI ESSAT DU 181079

MISE EN TENSION DU TIRANT 159a

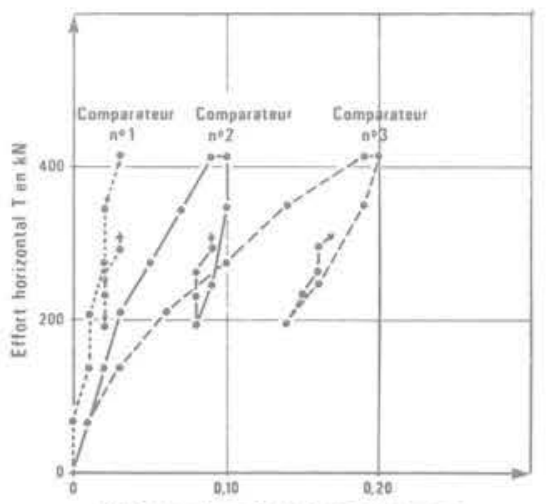

Déplacement au niveau du tirant $y$ en mm
LIILE . DEPLACEMENT DE LA PAROI ESSAI DU 18 1079

MISE EN TENSION DU TIRANT 160

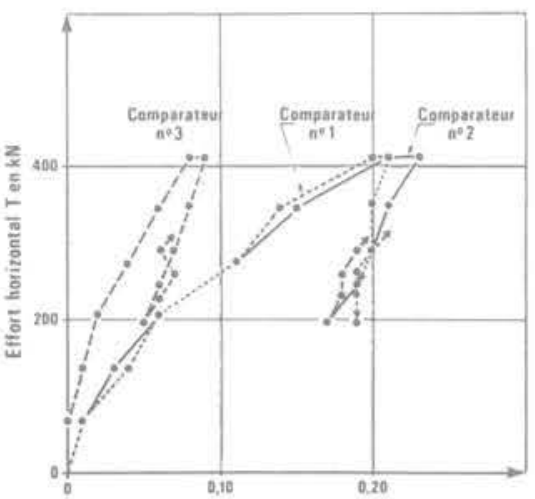

Déplacoment au niveau du tirant $y$ an mm
LHLLE DEPLACEMENT DE LA PARO ESSAI DU 1810.79

MISE EN TENSION DU TIRANT 161 .

Fig. 7. - Lille - Essai de chargement horizontal.

fig. $n^{\circ} 8$ ) de comparer l'ordre de grandeur du déplacement expérimental avec le déplacement théorique obtenu par résolution de l'équation classique:

$$
\text { EI } \frac{d^{4} y}{d z 4}+k_{B} y=0
$$

où $\mathrm{El}$ est le produit d'inertie de $1 \mathrm{ml}$ de soutènement et y le déplacement de la paroi à la profondeur $z$.

On obtient ainsi:

- sans appui en tête:

$$
\begin{gathered}
\frac{y}{T}=\frac{\gamma}{2 k_{B} \times B} \times\left[1+e^{-2 \gamma} \alpha \times\right. \\
(2+\cos 2 \gamma \alpha-\sin 2 \gamma \alpha)] \\
\text { avec } \gamma=\sqrt[4]{\frac{k_{B}}{4 E I}}
\end{gathered}
$$

T étant la composante horizontale de l'effort exercée par le vérin à la profondeur $\alpha$. Cet effort est diffusé horizontalement sur une largeur B supposée égale à la largeur du panneau sollicité.
Sollicitation

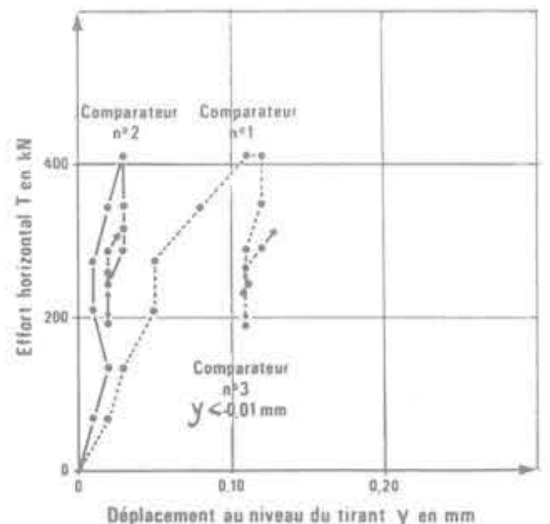

Déplacement au niveau du tirant $y \mathrm{en} \mathrm{mm}$

Réaction du sol

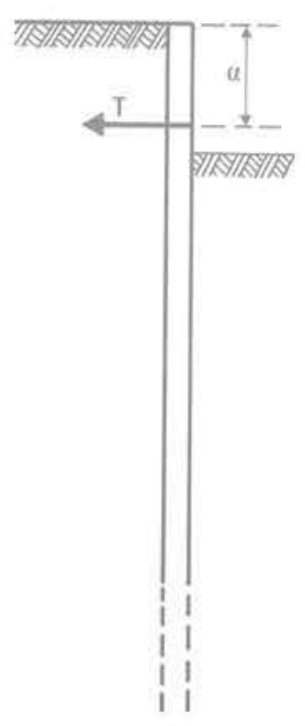

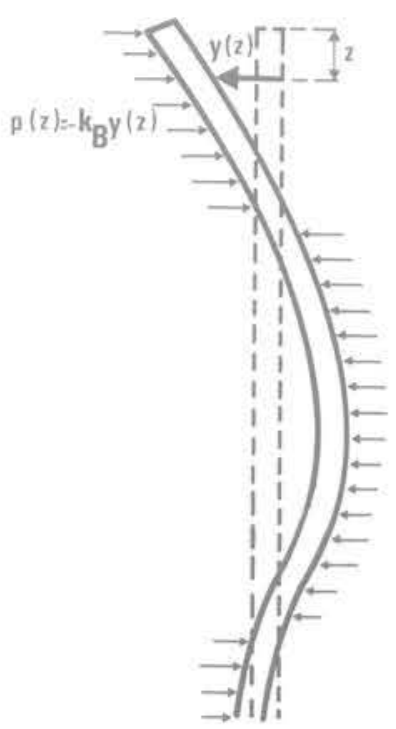

Fig. 8. - Paroi considérée comme une poutre sur appuis élastiques infiniment rapprochés. 


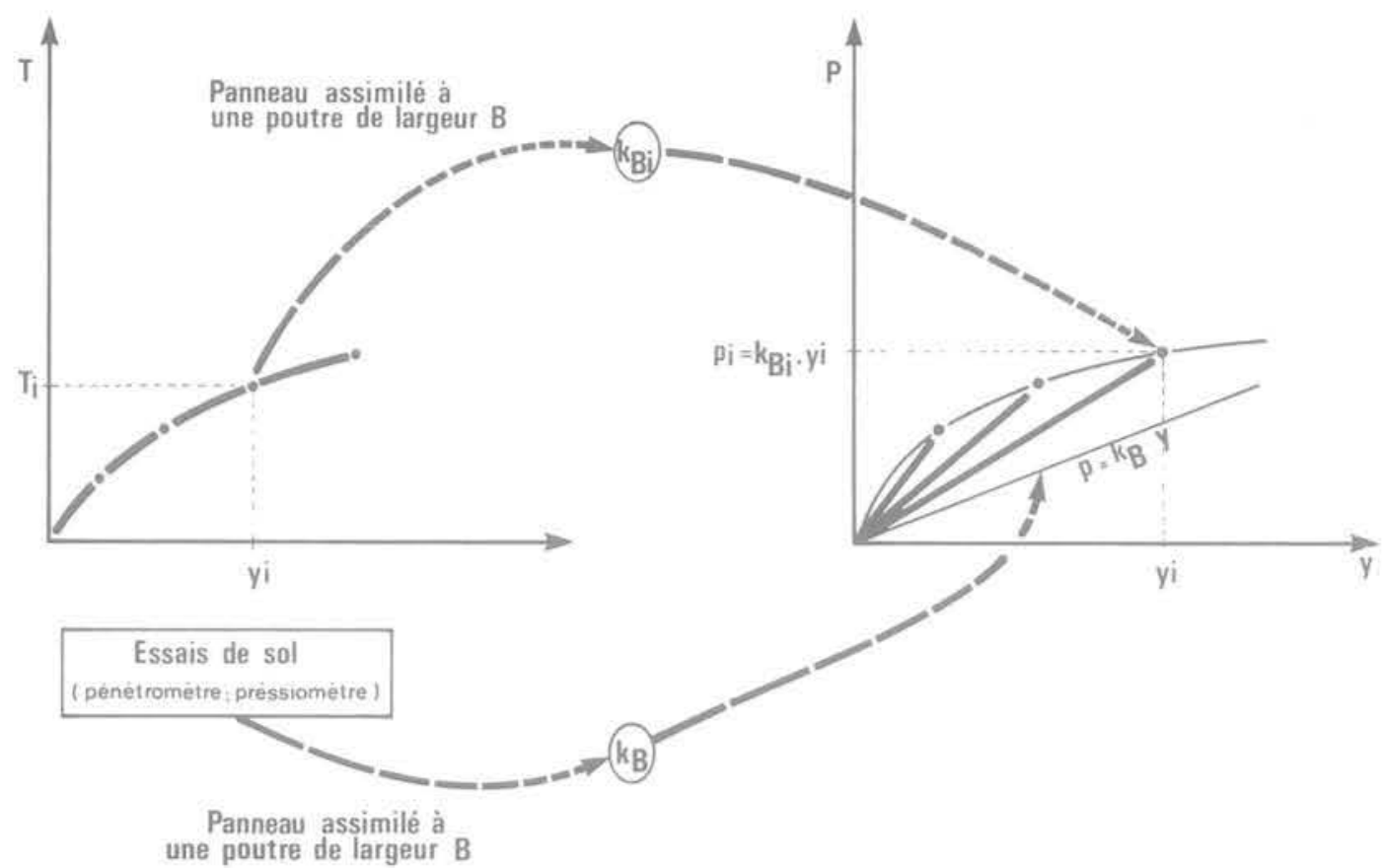

Fig. 9. - Approche "locale": principe de l'interprétation.

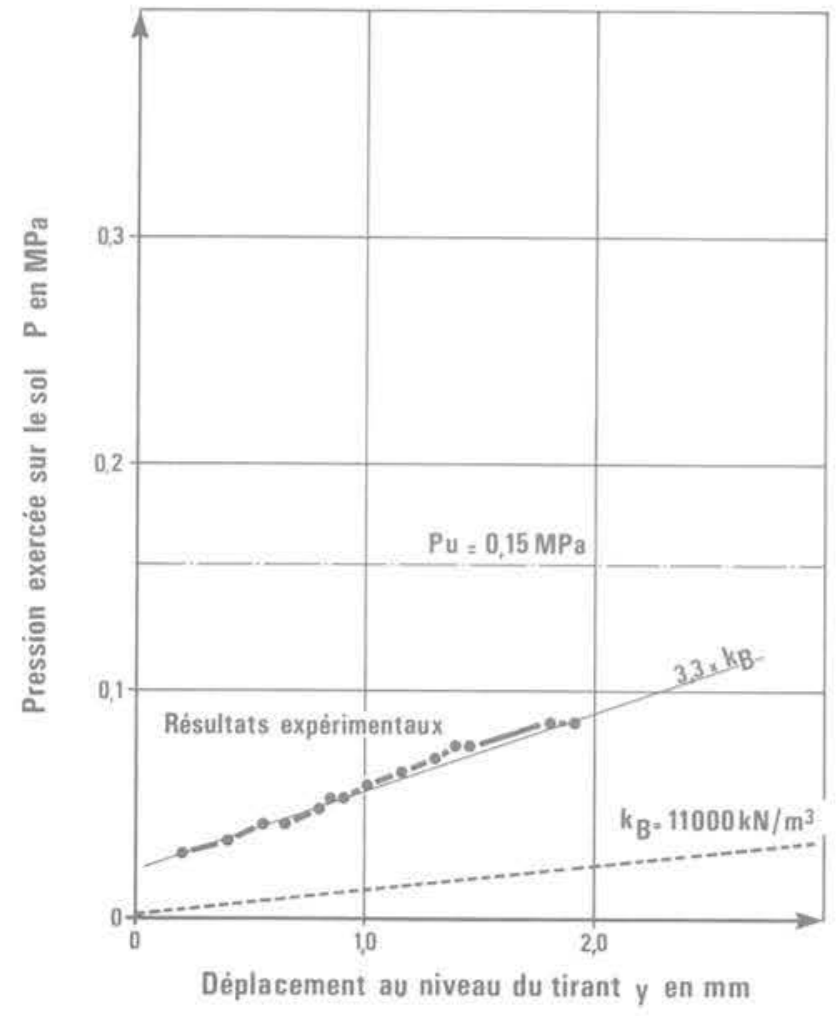

Fig. 10. - St-Prix - Comportement de l'interface sol-paroi.

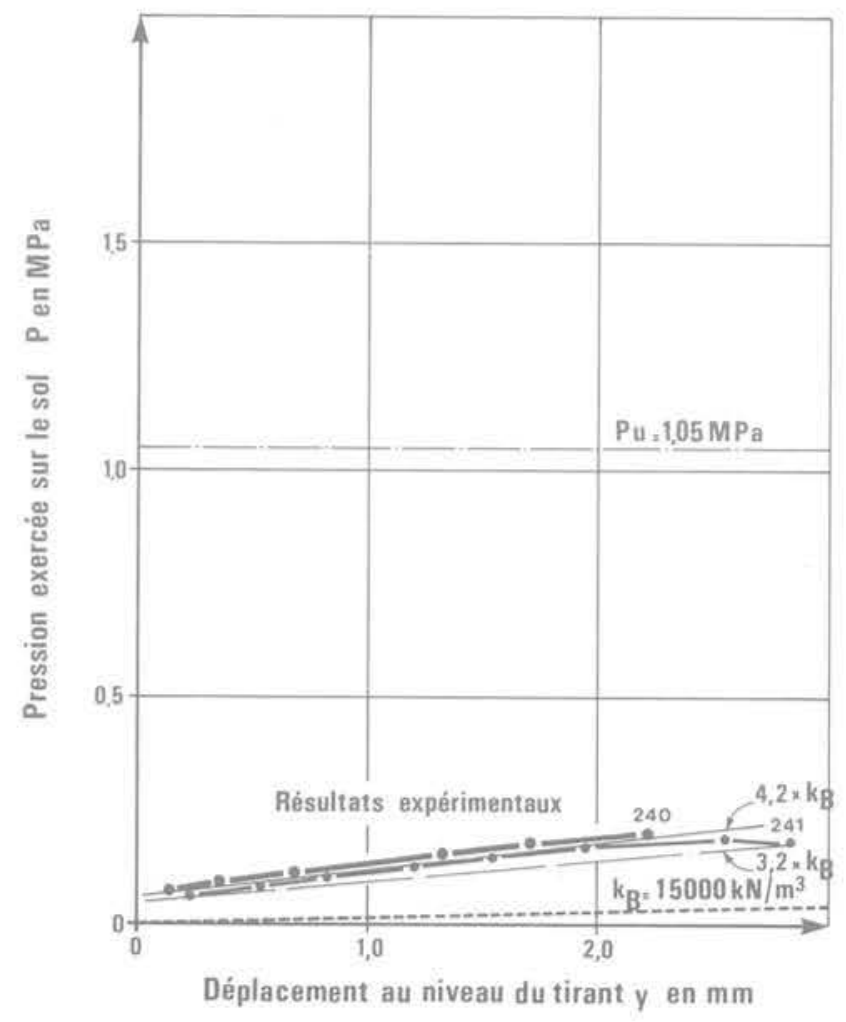

Fig. 11. - Lille - Comportement de l'interface solparoi. 
- avec appui en tête (cas d'une lieme):

$$
\begin{aligned}
& \frac{y}{T}=\frac{\gamma}{2 k_{B} \times B} \times\left[1-e^{-2 \gamma \alpha}\right. \\
& \times(\cos 2 \gamma \alpha+\sin 2 \gamma \alpha)]
\end{aligned}
$$

On vérifie que lerreur de principe dy/y inhérente à la méthode d'interprétation (assimilation d'un panneau de dimensions finies à une poutre semi-infinie, et nonprise en compte de la décompression élastique du terrain côté fouille) n'excède pas $25 \%$, ce qui est acceptable dans la mesure où l'on ne cherche qu'à comparer des ordres de grandeur.

Il est dès lors possible d'associer, à chaque couple ( $y_{i}$, $\mathrm{T}_{\mathrm{i}}$ ) de résultats expérimentaux, une valeur $\mathrm{k}_{\mathrm{B} i}$ du coefficient de réaction du sol, obtenue par résolution de l'une ou l'autre des équations implicites (5) et (6), et qui n'est autre que le module sécant au point $\left(y_{i}, p_{i}\right)$ de la courbe déplacement-pression caractérisant la réponse du sol au niveau du tirant (voir fig. $n^{\circ} 9$ ).

On constate en général que, la courbe expérimentale n'étant pas linéaire, le coefficient de réaction $k_{\mathrm{Bi}}$ diminue avec le déplacement atteint, ce qui met en défaut :

- le principe même de l'interprétation, qui caractérise le sol par un coefficient $k_{\mathrm{B}}$ unique alors que celui-ci varie avec le déplacement, donc avec la profondeur;

- et en fait le principe même du calcul des soutènements par la méthode du "coefficient de réaction

Toutefois, on constate systématiquement (voir les exemples indiqués sur les figures $n^{\circ} 10$ à 12) que, si

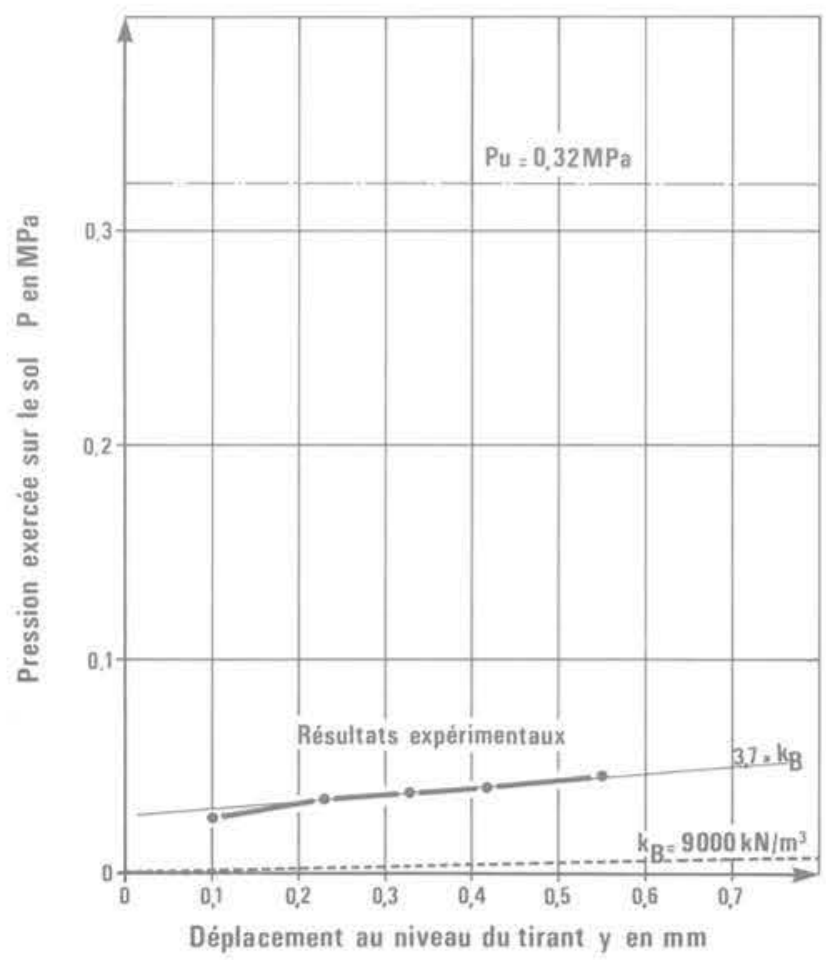

Fig. 12. - Gravelines - Comportement de l'interface sol-paroi. l'on fait abstraction de la partie de la courbe correspondant aux très faibles déplacements, il est possible de définir, pour le domaine de sollicitations considéré, un module tangent moyen caractérisant un comportement «pseudo-linéaire » du sol analogue à celui défini par Ménard pour l'essai pressiométrique.

Sur chaque figure sont représentées:

- la courbe pression-déplacement expérimentale, obtenue à partir des mesures brutes en assimilant la paroi à une poutre verticale sur appuis élastiques, de largeur B supposée égale à la largeur d'un panneau;

- la courbe conventionnelle $\mathrm{p}=\mathrm{k}_{\mathrm{BY}}$, le coefficient $\mathrm{k}_{\mathrm{B}}$ étant estimé à partir des essais de sol par les méthodes classiques (réf. 3 et 13, ou formules (2) et (3) en assimilant le panneau de paroi à une poutre de même largeur B.

On vérifie, en effet, au moyen des formules (5) et (6) que l'erreur $\frac{d(k B)}{k B}$ résultant d'une mauvaise connaissance de $\mathrm{B}$ reste comprise entre 0.1 et $0.3 \times \frac{\mathrm{dB}}{\mathrm{B}}$,

ce qui autorise à comparer les ordres de grandeur des coefficients de réaction expérimental et conventionnel pourvu qu'ils soient déterminés à partir d'une même valeur de $\mathrm{B}$.

On constate sur les différents sites étudiés (voir fig. $\left.n^{\circ} 13\right)$ que, si l'on exclut le domaine des très petites déformations*, le coefficient de réaction expérimental est du même ordre de grandeur, voire 2 à 4 fois

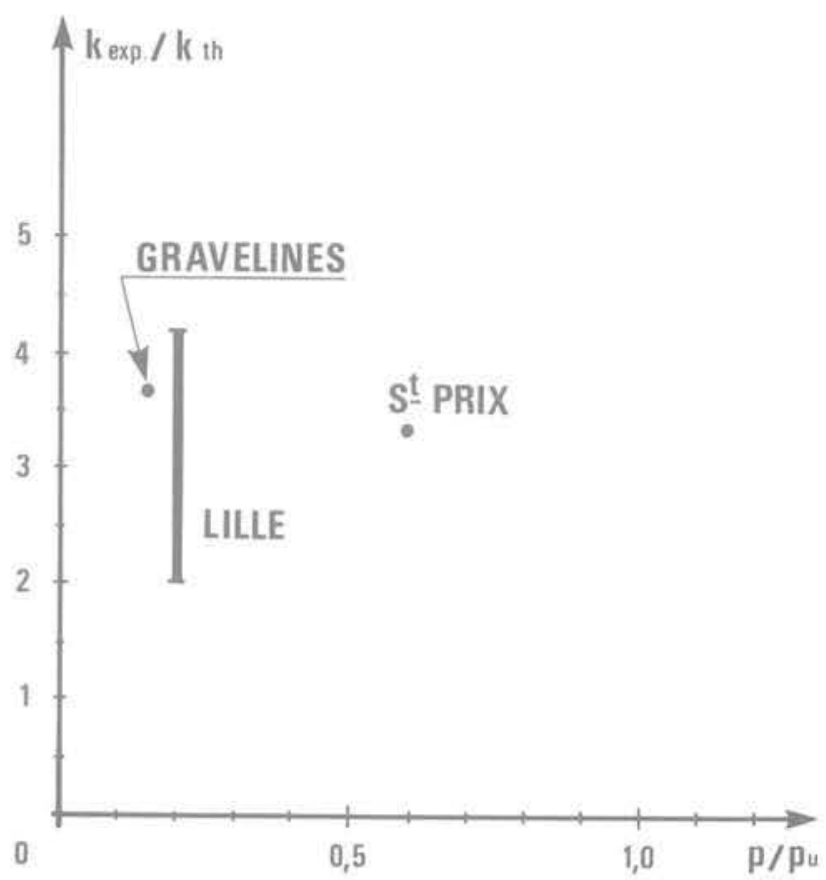

Fig. 13. - Résultats de l'étude "locale".

(*) Cas notamment des parois appuyées en tête sur une lieme où le rapport entre coefficients de réaction expérimental et conventionnel est de l'ordre de 20. 
supérieur au coefficient de réaction conventionnel pour un taux de sollicitation $\mathrm{p} / \mathrm{pu}$ (pu étant la pression maximale théorique correspondant à la mise en butée plastique du sol) inférieur ou égal à $50 \%$. Il est toutefois impossible de conclure de façon catégorique compte-tenu de l'incertitude inhérente à l'imprécision des mesures et de la méthode d'interprétation. Une étude complémentaire plus globale s'avère indispensable.

\section{APPROCHE «GLOBALE»}

Il s'agit ici d'analyser le comportement d'ensemble de l'ouvrage en service, à partir de mesures inclinométriques et topographiques permettant de connaître la déformée réelle de la paroi ou du rideau.

On a vu en effet que le calcul de la compression élastique du terrain par la méthode du coefficient de réaction suppose connue la largeur de la bande de terrain sollicitée par l'ouvrage, paramètre parfaitement défini dans le cas d'un pieu ou d'une semelle filante de largeur $\mathrm{B}$, mais inconnu a priori dans le cas d'un ouvrage de soutènement, où la hauteur D de terrain réellement comprimé ne peut être connue qu'expérimentalement, par exemple en mesurant la déformée.

Partant de ce principe, l'étude a été conduite suivant la méthode indiquée sur la figure $\mathrm{n}^{\circ} 14$ : l'examen de la déformée expérimentale permet de définir une ou plusieurs hauteurs Di correspondant par exemple, pour une phase de terrassements donnée, à la zone sollicitée par les tirants précontraints et à la mise en butée (généralement élastique) du sol sous le fond de fouille. Chaque hauteur $\mathrm{Di}$ permet de calculer, à partir de l'une ou de l'autre des méthodes classiques, un coefficient de réaction $\mathrm{k}_{\mathrm{Di}}$ dit conventionnel.

Parallèlement, l'interprétation de l'ensemble des mesures réalisées sur l'ouvrage (déformée, tension des tirants, contraintes dans le béton et/ou l'acier, pressions de contact sol-paroi, etc.) permet généralement de déterminer, la ou les valeurs du coefficient de réaction qui permettent de rendre compte au mieux de la réalité expérimentale.

On dispose donc, là encore, de valeurs expérimentales et conventionnelles du coefficient $\mathrm{k}$ qu'il est intéressant de comparer.

On trouvera sur les fig. $n^{\circ} 15$ à 21 les résultats relatifs à des chantiers réalisés par Soletanche d'une part, à diverses instrumentations dont les résultats publiés permettent l'interprétation décrite ci-dessus d'autre part.

Les valeurs conventionnelles du coefficient $k_{D}$ ont été déterminées à partir d'essais pressiométriques, par application de la formule (1) dans le cas général où la déformation de l'écran intéresse la surface libre du massif de sol, par application de la formule (2) dans le cas contraire (exemple fig. $\mathrm{n}^{\circ} 16$ ). Compte tenu de

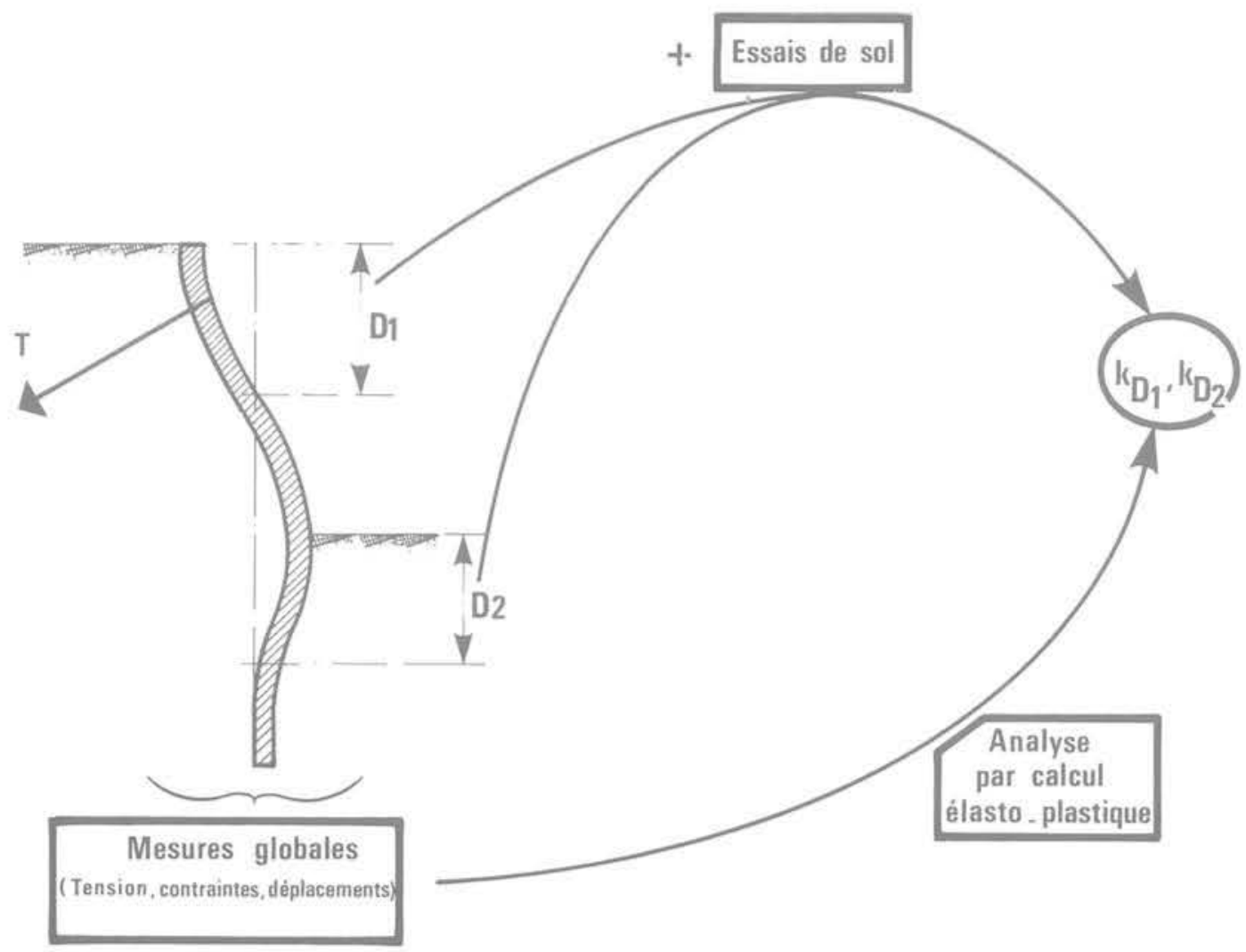

Fig, 14. - Approche "globale". Principe de l'interprétation. 


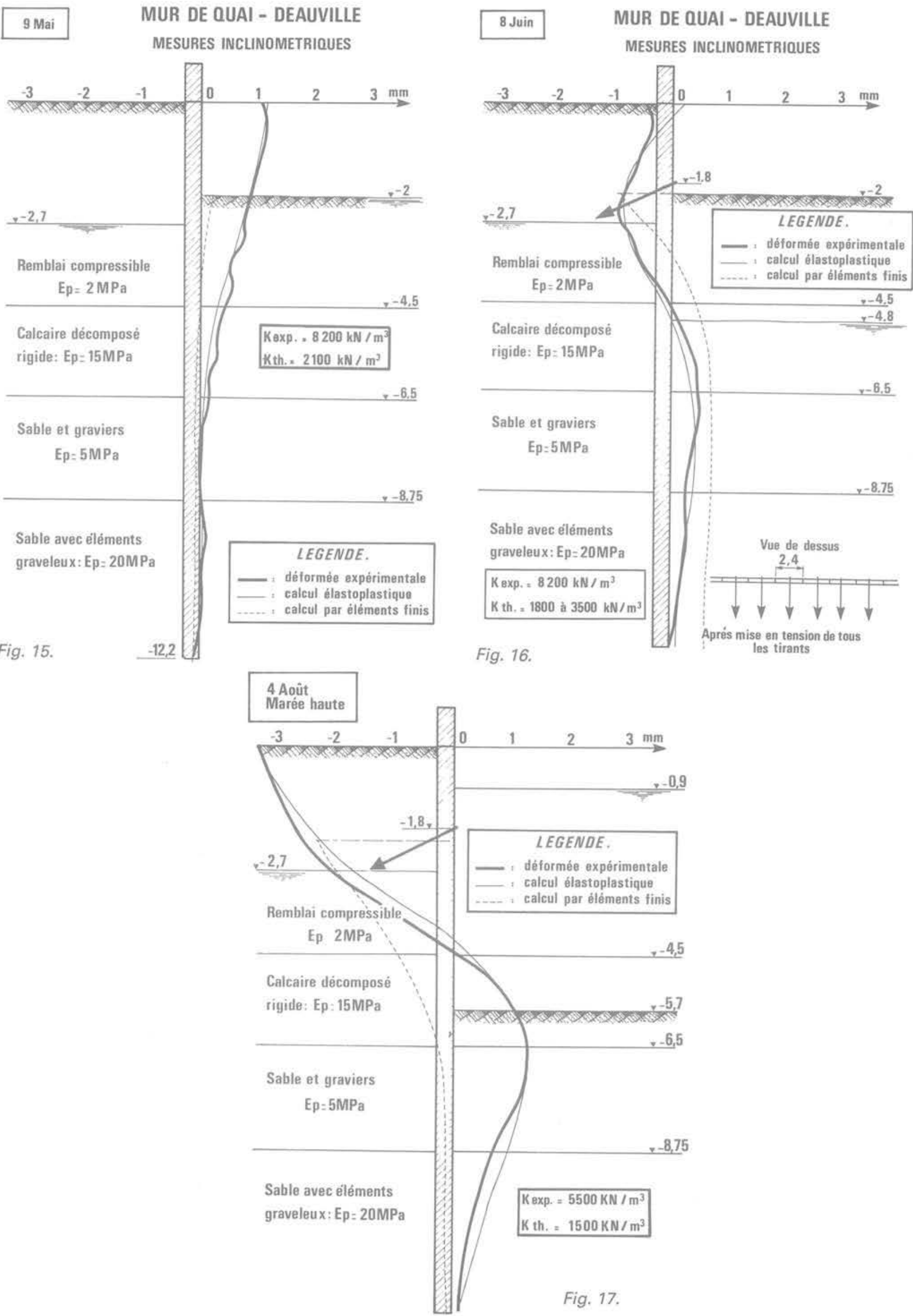

Fig.15 - Fig. 16 - Fig. 17. - Deauville - Déformée de la paroi. 


\section{METRO DE LILLE \\ DEFORMATION DU RIDEAU DE PALPLANCHES}

Tube inclinométrique $\mathrm{N}^{\circ} 4$

Etat initial : 20-3-81

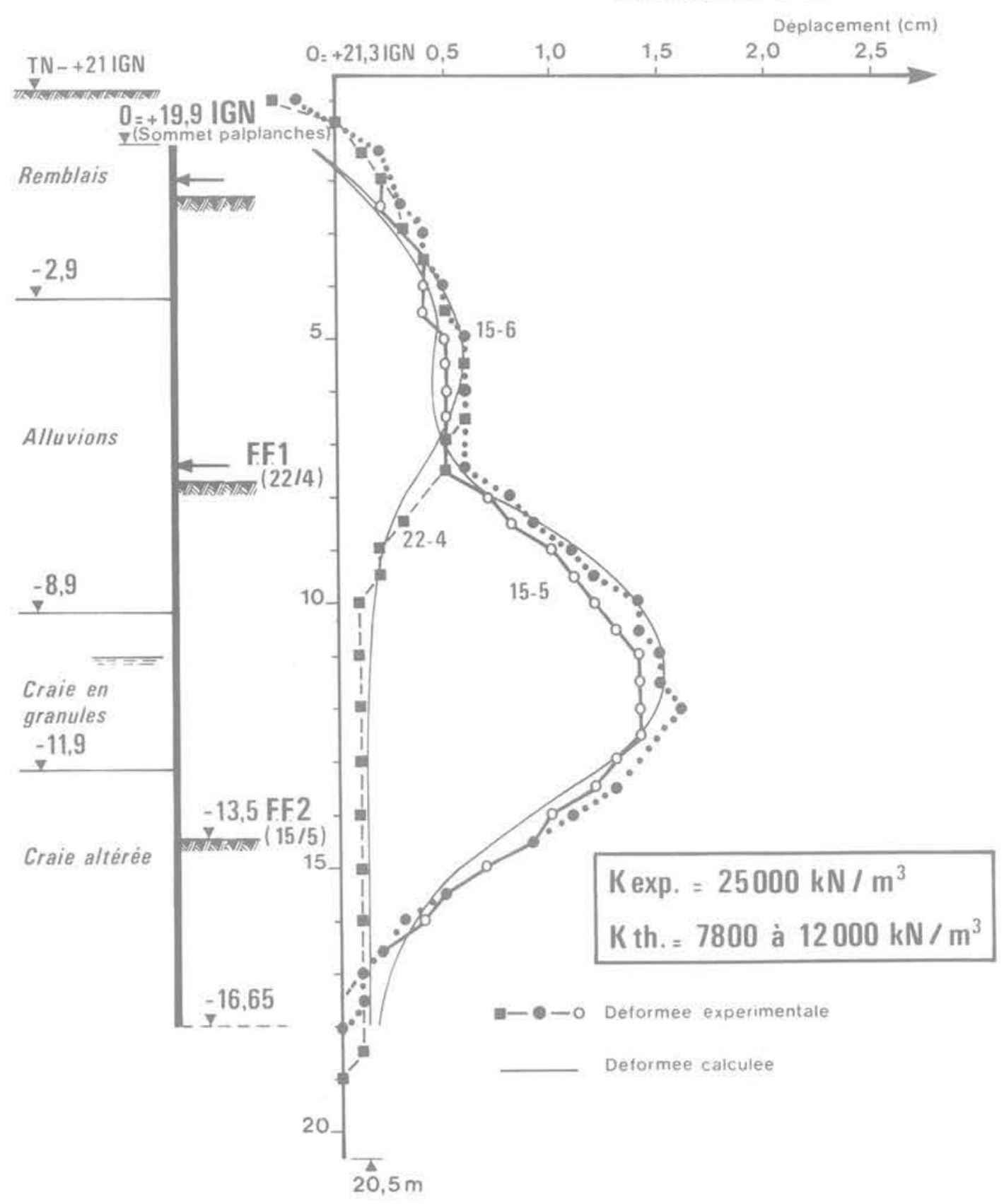

Fig. 18. - Lille - Déformée du rideau. 


\section{QUAI A MOULINEAUX}

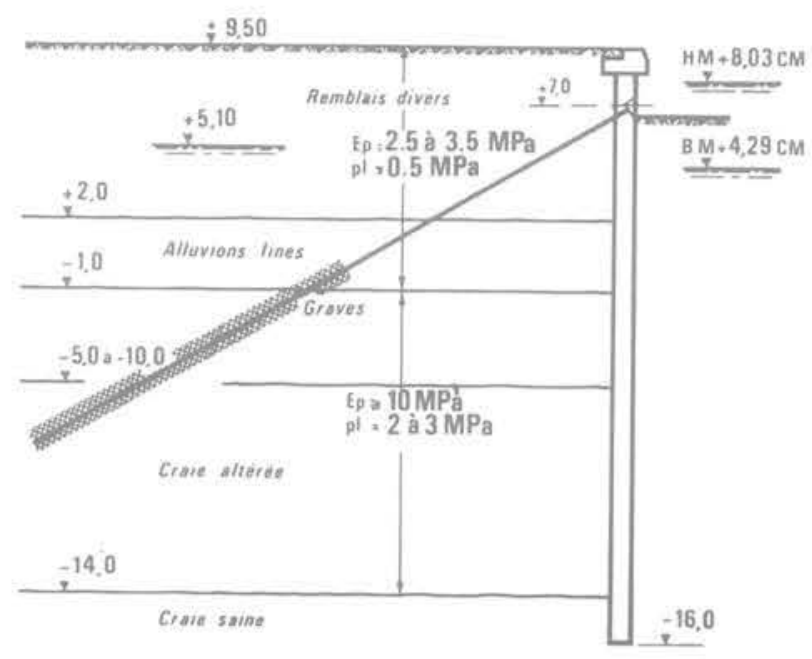

$K \exp ,=2500 \mathrm{kN} / \mathrm{m}^{3}$

$K$ th. $\leqslant 1300 \mathrm{kN} / \mathrm{m}^{3}$

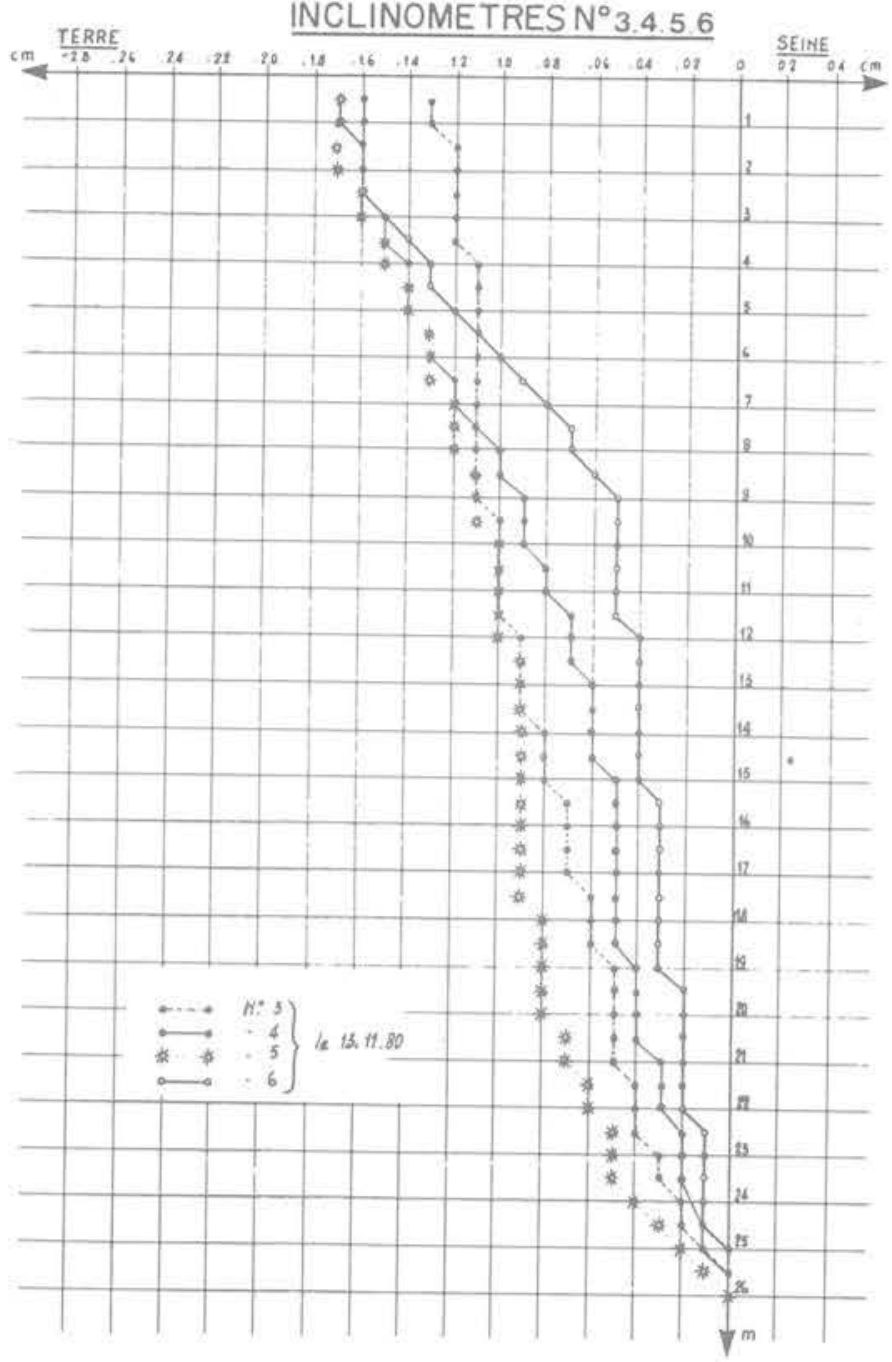

Fig. 19. - Rouen - Déformée de la paroi.

PORT DU HAVRE

DEFORMATIONS DE LA PAROI MOULEE (d après Blivet, Bonafous, Frank, Josseaume)

$$
\text { Date : } 1981
$$

$0 \quad 5 \quad 10 \quad 15(\mathrm{~mm})$

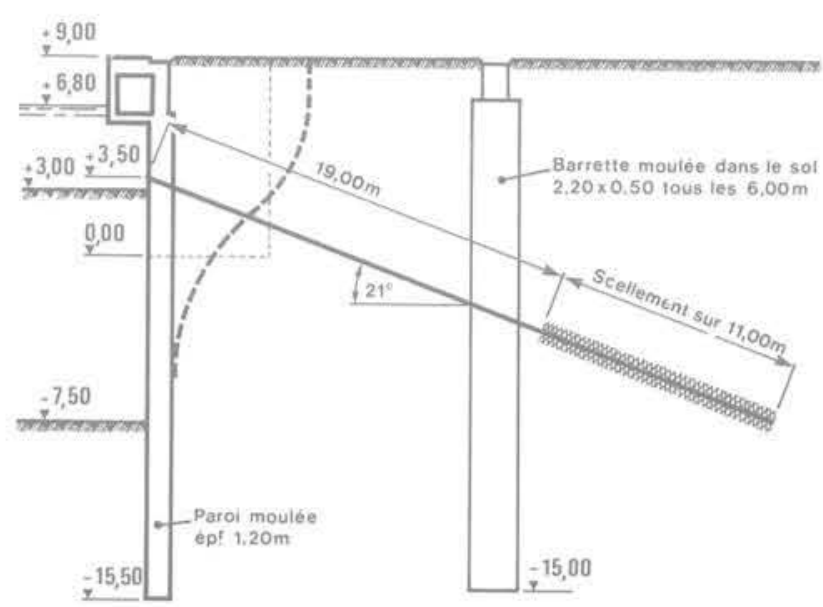

Kexp. moyen $=15000 \mathrm{kN} / \mathrm{m}^{3}$ K th. $=3500 \mathrm{kN} / \mathrm{m}^{3}$

Fig. 20. - Le Havre - Déformée de la paroi.
PUTEAUX

DEFORMATION DU RIDEAU DE PALPLANCHES (d'après Balay, Bonafous, Frank, Gigan , Josseaume)

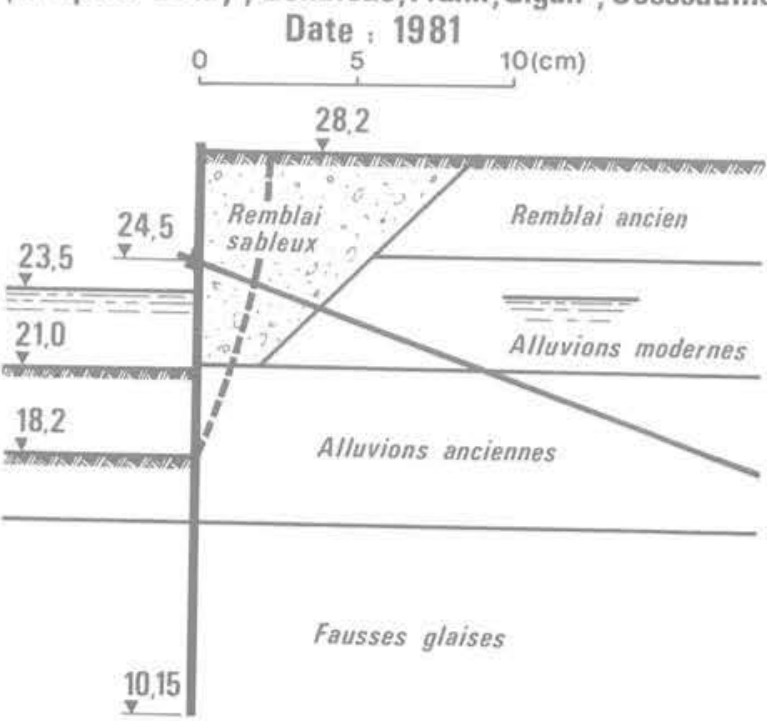

Kexp. moyen $=2500 \mathrm{kN} / \mathrm{m}^{3}$
$\mathrm{Kth} .=1200 \mathrm{kN} / \mathrm{m}^{3}$

Fig. 21. - Puteaux - Déformée du rideau. 


\section{LYON}

\section{DEFORMATION DU RIDEAU DE PALPLANCHES (d après Josseaume, Gigan, Ferrand, Houy ) \\ Date : 1977 \\ o $1020(\mathrm{~mm})$}

$$
0,0
$$
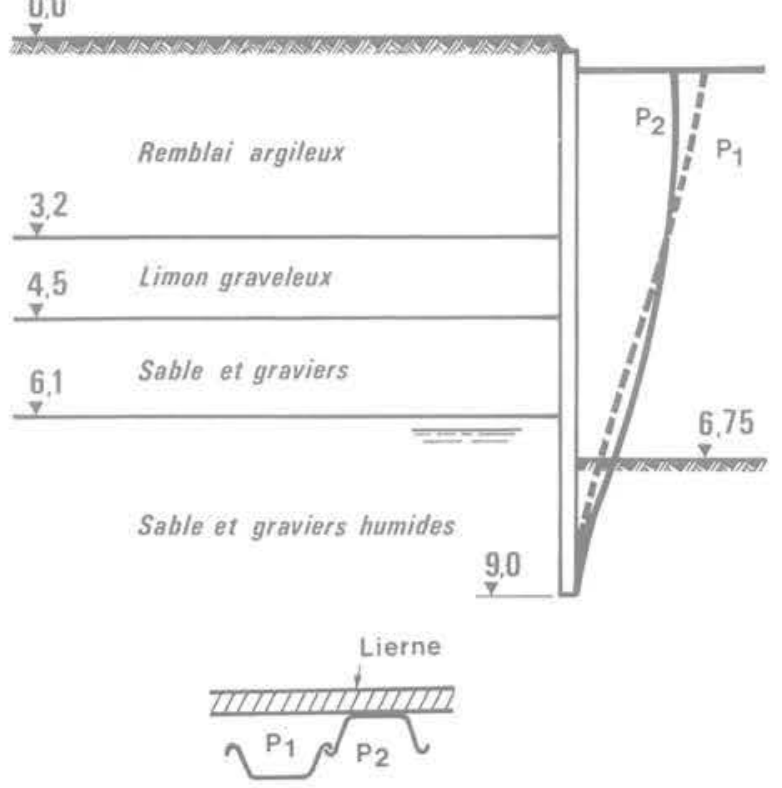

$$
\begin{aligned}
& K \exp .=70000 \mathrm{kN} / \mathrm{m}^{3} \\
& K \text { th. }=70000 \mathrm{kN} / \mathrm{m}^{3}
\end{aligned}
$$

Fig. 22. - Lyon - Déformée du rideau.

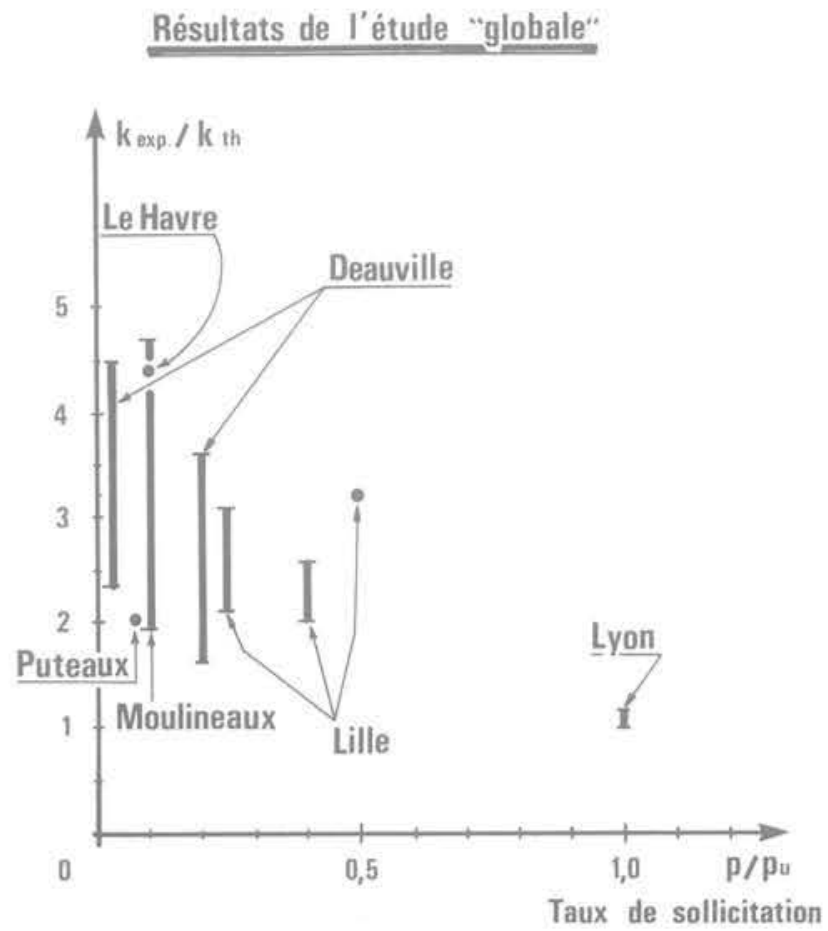

Fig. 23. - Résultats de l'étude "globale». l'incertitude inhérente à la mesure de déformée, la hauteur D prise en compte ne correspond qu'aux déplacements de l'écran jugés significatifs, c'est-à-dire supérieurs à $20 \%$ du déplacement maximal; on tend ainsi à surévaluer le coefficient de réaction conventionnel en minimisant $D$.

L'ensemble des résultats, reproduit sur la figure $n^{\circ} 23$ met en évidence pour l'ensemble des sites étudiés un rapport entre coefficients de réaction expérimental et conventionnel compris entre 2 et 4 pour des taux de sollicitation $\mathrm{p} / \mathrm{pu}$ n'excédant pas $50 \%$, ce qui confirme donc les résultats de l'approche "locale».

\section{CONCLUSION}

La première conclusion de cet ensemble d'études expérimentales est que les méthodes de calcul utilisant la notion de "coefficient de réaction " permettent, en dépit d'hypothèses de base très simplificatrices, de rendre compte de façon très correcte du fonctionnement des ouvrages de soutènement souple.

La détermination d'un ordre de grandeur raisonnable du coefficent $k$ est possible a priori, sous réserve de tenir compte:

- de la hauteur approximative du terrain comprimé, qui dépend, notamment, de la rigidité relative du soutènement par rapport au sol. Elle peut être soit estimée, ce qui est le cas général, soit déterminée par itérations:

- de la non-linéarité de la réaction du sol. A cet égard, la présente étude conduit à proposer la loi schématisée sur la fig. $n^{\circ} 24$ qui tient compte de la

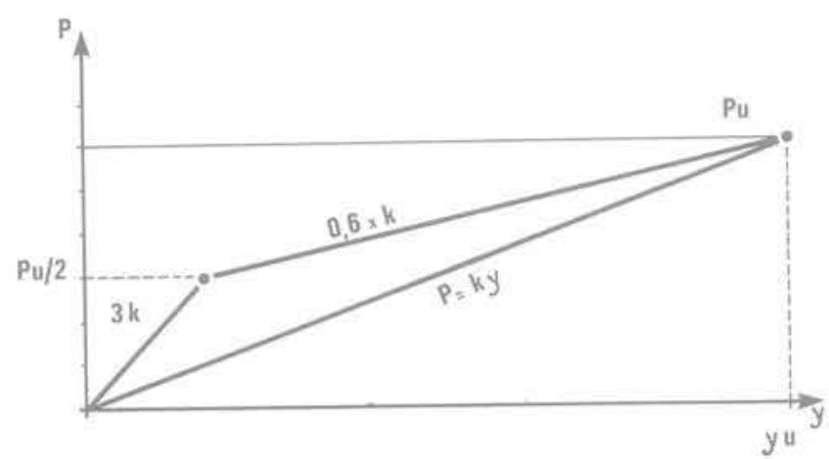

Fig. 24. - Comportement expérimental de l'interface sol-écran.

raideur plus importante observée dans le domaine des faibles déplacements. La prise en compte d'une loi de ce type, plus conforme à la réalité, conduit à un réajustement des efforts (moments fléchissants et efforts tranchants) de l'ordre de 5 à $30 \%$ en règle générale.

Il faut toutefois noter que deux difficultés importantes liées à l'emploi des méthodes de calcul élastoplastiques n'ont pas été abordées. Ce sont: 
- la détermination du coefficient de poussée des terres au repos, Ko, qui dépend également de paramètres liés au projet et notamment du bétonnage dans le cas d'une paroi moulée (réf. 12);

- l'impossibilité d'apprécier correctement les déplacements d'ensemble du massif de sol situé derrière le soutènement, notamment dans le cas d'une parol ancrée par plusieurs niveaux de tirants où l'on assiste au développement d'un "effet-gabion". Ces déplacements d'ensemble ne peuvent être appréciés qu'au moyen d'un calcul par la mêthode des éléments finis, ou plus grossièrement par un calcul de résistance des matériaux (réf. 6). Les calculs utilisant le coefficient de réaction sont donc mieux adaptés à la détermination des efforts qu'à celle des déplacements d'ensemble.

Par contre, on signalera enfin que la méthode trouve une application particulièrement intéressante dans le domaine des parois moulées circulaires de grand diamètre, où les efforts de flexion verticale peuvent être calculés à l'aide du coefficient de réaction d'un terrain fictif simulant la rigidité de l'anneau comprimé (voir fig. $\mathrm{n}^{\circ} 25$ ).

On peut donc conclure que le coefficient $\mathrm{k}$, en dépit de sa nature empirique et des difficultés rencontrées

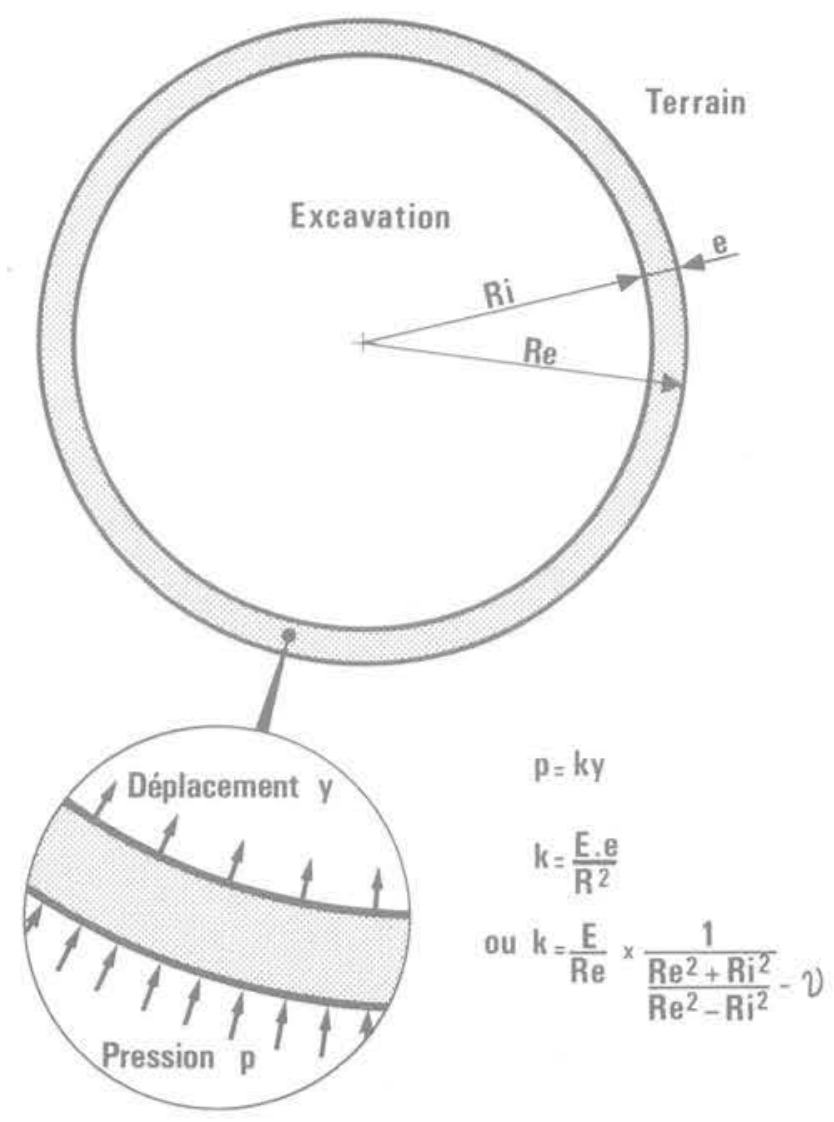

Fig. 25. - Calcul des parois circulaires. lors de sa détermination, constitue actuellement un outil de travail particulièrement performant pour le calcul des efforts sollicitant les ouvrages de soutènement souples.

L'auteur tient à remercier MM. GOUVENOT et GAMBIN pour les consells dont il a bénéficié au cours de cette étude.

\section{RÉFÉRENCES BIBLIOGRAPHIQUES}

1. BALAY, BONAFOUS, FRANK, GIGAN, JOSSEAUME (1981). - Expérimentations de soutènement ancrés par tirants actifs. - $10^{\mathrm{e}}$ ICOSOMEF, Stockholm, tome 2, p. 29.

2. BLIVET, BONAFOUS, FRANK, JOSSEAUME (1981). - Comportement d'un quai en paro moulée au port du Haure. - Bull. Liaison L.P.C. $\mathrm{n}^{\circ} 113$, mai-juin 1981.

3. CAMPUS (1972). - Contribution à l'étude des pièces fléchies dans le sol. Application aux pieux et aux palplanches. - Mémoires CERES de l'Université de Liège, n³9, auril 1972.

4. CAQUOT-KERISEL (1966). - Traité de mécanique des sols. $-4^{e}$ édition, Ed. Gauthier-Villars.

5. COURBON (1971). - Résistance des matériaux. Tome 2, Ed. Dunod.

6. GOUVENOT, BUSTAMANTE (1979). - Mesures in situ sur les ouvrages maritimes de soutènement. - Annales I.T.B.T.P., n 375 , Série Sols et Fondations, $n^{\circ} 167$, septembre 1979.

7. JOSSEAUME, GIGAN, FERRAND, HOUY (1977). - Mesures et observations sur rideaux de palplanches. - Colloque palplanches Maîtres d'CEuvreProducteurs, Paris, avril 1977.

8. LACROIX, MARSH (1972). - Stabilité de culées. - Revue Canadienne de Géotechnique, Vol 9-1. p. 1-24.

9. MENARD (1961). - Influence de l'amplitude et de l'histoire d'un champ de contraintes sur le tassement d'un sol de fondation. $-5^{\mathrm{e}}$ ICOSOMEF, Paris, tome 1, p. 249-253.

10. MENARD, BOURDON (1965). - Calcul des rideaux de soutènement. Méthode nouvelle prenant en compte les conditions réelles d'encastrement. Sols-Soils n¹2, mars 1965.

11. MENARD (1973). - Règles d'utilisation des techniques pressiométriques, contraintes et déformations dans un pieu soumis à des efforts horizontaux. Notice spéciale $n^{\circ}$ 2D/62/69. Documentation *Techniques L. Ménard».

12. REYNAUD, RIVIERE (1981). - Mesure des pressions développées dans une paroi moulée en cours de bétonnage. - Bull. Liaison L.P.C., n 113, mai-juin 1981.

13. TERZAGHI (1955). - Evaluation of coefficients of subgrade reaction. - Geotechnique, vol. 4 